\title{
Posture Monitoring of Basketball Training Based on Intelligent Wearable Device
}

\author{
Zengyu $\mathrm{Ma}^{1}$ and Qi Hao $\mathbb{D}^{2,3}$ \\ ${ }^{1}$ Sports Department, Northeastern University, Shenyang 110167, China \\ ${ }^{2}$ School of Sports and Health, Linyi University, Linyi 276000, China \\ ${ }^{3}$ Department of Leisure-Sports, Pai Chai University, Daejeon 35345, Republic of Korea
}

Correspondence should be addressed to Qi Hao; haoqi@lyu.edu.cn

Received 2 December 2021; Accepted 7 January 2022; Published 12 February 2022

Academic Editor: Deepak Kumar Jain

Copyright (C) 2022 Zengyu Ma and Qi Hao. This is an open access article distributed under the Creative Commons Attribution License, which permits unrestricted use, distribution, and reproduction in any medium, provided the original work is properly cited.

\begin{abstract}
The relevant content of China's sport work clearly pointed out that it is necessary to improve the physical and mental health of the citizens. Combining the current development hotspots of mobile smart terminals and smart wearable devices, smart wearable devices are analyzed from the function and development history in order to find an effective combination of smart wearable devices and sports work. This article describes the youth as the main body receiving grassroots physical education of nonsports special students and basketball enthusiasts. In the process of physical education teaching, the problem of pain points is more prominent because most teenagers in the basketball education at the grass-roots level do not have the opportunity to get one-toone private counseling. When there are problems in the operation, students cannot be guided to conduct training or practice by themselves. This paper aims to meet the demand of real-time monitoring of dribbling posture in basketball dribbling training and proposes a low-cost product solution to help teenagers carry out basketball dribbling training by themselves: intelligent wearable product of head wrist and dribbling assistant DribbleAid, which is used to monitor common bad posture problems of users in dribbling training and give corresponding reminders to deal with users' pain points. In this work, various experiments have been carried out for the proposed method and system. A large number of experimental results show that the method designed in this paper can effectively monitor the basketball training posture.
\end{abstract}

\section{Introduction}

The adolescents in this study are mainly non-sports-specialized students and basketball enthusiasts who are taking adolescents as the main body and are receiving grassroots physical education. The so-called grassroots physical education refers to the physical education carried out by grassroots schools for nonsports students, including primary school, junior high school, high school, and high school nonsports specializing students. In addition to the previously mentioned population, this study also included the non-physical-college students who are receiving college physical education into the target group. China has always attached great importance to the improvement of students' comprehensive quality in order to promote the overall development of students' morality, intelligence, and physical fitness. However, due to the impact of exam-oriented education, there is a shortage of physical education teachers in grassroots schools, resulting in a relatively low quality of physical education in grassroots schools. Nowadays, primary and middle school physical education teachers or coaches often become unable to guide students in training because of the excessive number of students. It is reflected from the side that, in China's grassroots physical education, the teacherstudent ratio is even less than $1: 30$. This indirectly reflects the shortage of coaches in youth dribbling training. If the teacher-student ratio is too low, the coach or physical education teacher will not be able to fully guide or take care of each student. If the bad posture in training is not corrected for a long time, it will develop into a bad habit. It will be 
difficult to change in the future. For example, the habit of looking down at the ball while dribbling is one of the common bad habits in Chinese basketball courts, and it is difficult to change. If you can develop the habit of raising your head to dribble during the enlightenment stage of youth basketball, it will greatly improve the overall dribbling ability of Chinese basketball players or enthusiasts. In addition, in the process of personal training, the cost of oneon-one basketball teaching is relatively large. Therefore, it is difficult to effectively correct the training habits of personal training, the positive effects of training are not obvious, and the efficiency is low [1-7].

Smart wearable devices have a long history of development. In the 1960s, considerable ideas and prototypes were shown. Devices with smart wearable styles emerged in the 1970s and 1980s. Steveman's Apple II 6502 computer prototype wearable computer research and development is one of the representatives. Smart wearable devices are accompanied by computer software and hardware standardization and the rapid development of the Internet, and the styles of the devices are presented in a variety of ways. Important research value has been shown gradually in industry, medical, military, education, entertainment, and many other potential applications. The rapid development of smart wearable devices has quickly integrated into all aspects of life, and it marks the deepening of artificial intelligence. Not only can people use smart wearable devices to explore their own and external information, but also they can process internal and external information more efficiently with the help of computers, enabling more seamless communication. The application fields of smart wearable devices can be divided into two categories: in vitro evolution field and self-quantification field. In the field of in vitro evolution, this type of smart wearable device has the ability to help users improve the perception and processing capabilities of obtaining information. Its application fields are extremely wide, from entertainment and leisure or information exchange to the overall application of the industry. Users can strengthen and improve their own skills through smart wearable devices with various functions. Among the important participants are smart watches or smart glasses in major high-tech companies instead of relying on other external devices such as mobile phones to complete the function of interacting with users. In the field of selfquantification, the more common ones are divided into two major application fields: medical care and outdoor fitness. Prior to this, manufacturers were mainly involved in the detection and processing of medical portable equipment, blood pressure, heart rate, and provision of medical signs programming, in more diverse forms, including medical clothing and implanted chips. For the latter, manufacturers are mainly involved in outdoor sports and some start-ups. Light portable bracelets and watch accessories are taken as the main form, physical exercise, or outdoor data such as energy consumption. The monitoring analysis and service of heart rate, frequency, pressure, diving depth, and altitude indicators are realized. The mainstream product forms of smart wearable devices include wrist-supported watch, footsupported shoes, head-supported glass, and various nonmainstream products such as smart clothing, school bags, crutches, and accessories [8-15].

The contribution for this work is as follows: (1) It identified the pain points in the youth basketball dribble training scene. (2) It completed the more systematic conceptual design of DribbleAid. (3) The determination methods and related algorithms of dribbling over wrist, dribbling speed too slow, head down time too long, and nondominant hand weak in different dribbling events have been determined.

\section{Related Work}

There were relatively many related researches on basketball's sports posture and evaluation and researches on a variety of characteristic factors related to basketball's sports posture. Literature [16] analyzed the risk factors of ankle injury by evaluating the posture swing of high school basketball players on the court. 42 high school athletes who had no history of lower limb injuries before the start of the season were selected, and a physical therapist conducted biomechanical tests on them, including the strength of the ankle joint for uniform motion, the joint force of the one-legged standing swing, and the flexibility of the curved back. Then, every month, the health of each player was investigated by e-mail. The incidence of ankle injuries that occurred during the season as expected was recorded. The results of these preseason measurements were analyzed to correlate whether any of these measured variables can predict future ankle injuries. Literature [17] carried out a research in order to get the best defensive posture in basketball. The best hip and knee angulation was determined by the lateral speed and reaction speed of 18-23-year-old women's basketball players in different postures. In the experiment, the total body reaction time, muscle reaction time, latency, and maximum floor reaction force estimated from the vertical force signal were measured, as well as the first step width and initial speed of the torso estimated from two-dimensional video analysis. These results provided clear evidence that the 30degree and 30-degree hip and knee postures have the advantages of the initial speed of the body shaft and the maximum floor reaction force when walking sideways. Literature [18] investigated the coordination of a wholebody task, where shooting posture and ball movement trajectory were the independent variable and dependent variable, and the posture stability of the shooter and the posture movement and the release of the ball could be highly reliable predictors. A function of shooting posture, ball shooting level, and hit rate was got. Literature [19] studied the footwork of basketball players with a history of tibia injuries. The conclusion found that foot abnormalities were common in basketball players with a history of foot and ankle diseases. These findings could support the idea that both shoes and orthopedic prescriptions can prevent and treat basketball players' tibia injuries. Literature [20] studied the influence of the position of the defender and the angle relationship of the two-tuple on the decision-making process of the attacker in the basketball 1v1 subsystem. After the video recording performance behavior, the positions of the 
attacker's and defender's feet and the movement trajectory of the participant's displacement were obtained through digital analysis. It turned out that the defender's posture was related to the decision of the attacker's driving side, and the interpersonal distance was scaled for each two-tuple. When the attacker detects an alignment between the defender and the basket, the two-tuple and the basket need to be repositioned.

Wearable devices had a relatively long history and had experienced the development process from gaming tables to ordinary consumers [21]. They could be traced back to the middle of the last century, and the concept of wearable computing was first proposed by literature [22]. Literature [23] invented a wearable computer to profit from the gambling game of blackjack, and a large number of wearable applications and technologies were used on the gaming table until the end of the 1970s. As this concept slowly became popular, people began to apply wearable devices in the medical field in the 1980s to help people with hearing impairments. Until the first commercial head-mounted display Private Eye [24] appeared, more and more solutions were proposed. At the same time, the development trend had begun to quietly point to the needs of daily life, and more and more applications for daily life had been developed. This was due to people's constant pursuit of convenience in life. Another development trend of wearable devices is accessibility; that is, people want to use such devices anywhere and anytime to make their lives more convenient. In 1984, the movie Terminator was released in theaters. The holographic imaging technology used in the movie plot to present real-world scenes from the perspective of a robot with a semimechanical structure was today's holographic imaging technology. Several years after the document in [25] invented the wearable device of blackjack, the document in [26] invented a wireless device to upload images to the network, which was regarded as the earliest life recorder. Literature [27] started from the interaction between the human body and wearable devices and conducted in-depth research on how wearable devices in motion could better interact with users. The principles of wearable device design in product form and choice of wearing position were obtained. Literature [28] conducted an in-depth discussion on the timeliness of visual warnings of wearable devices worn on different parts from the perspective of visual information transmission. Literature [29] studied the interactive characteristics of wearable devices in supporting task completion and physical experience from the perspective of human factors engineering. From the perspective of perception and cognition, literature [30] suggests that wearable devices can help users get new cognition due to forgetting. A lot of exploration had been carried out in the interactive design of wearable devices abroad. At the actual application level, in 2014 and 2015, Google, Apple, and other companies also designed their own application platforms accordingly, and they proposed the interaction criteria for smart wearable devices. Luke Wroblewski, as a leader in foreign interaction designers, had more prominent and in-depth research on smart watches [31].

\section{Method}

3.1. Product Form of DribbleAid. Through analysis, it is found that there are two general product forms of intelligent wearable devices: one is pure hardware device, and the other is the combination of hardware device and mobile device APP. Both forms have their own advantages. The combination of hardware devices and terminals is a popular product form in the market at present. Most terminals are personal mobile devices-mobile phones, such as Jordan basketball shoe chip and its exclusive APP, and Xiaomi wristband and its exclusive APP. Its advantages are obvious. With terminals, users can fully improve their operating experience by virtue of their computing and display capabilities as well as richer interaction modes, and the data information that is not easily perceived can be visualized through augmented reality.

Through the above analysis, the product form of DribbleAid is finally determined to be "a combination of hardware and mobile APP." It can not only get the maximum satisfaction in the function but also ensure that most users can enjoy a higher product experience. DribbleAid's hardware part has a certain computing function and can carry out a small amount of computing and storage functions such as posture monitoring and record historical training data; data visualization function is relying on the mobile APP. The advantage of this idea is that the posture monitoring function, which is frequently used, can still work normally when there is no mobile phone or it is not suitable to use mobile phone.

3.2. Process Design. The entire process logic of DribbleAid is divided into two lines, the training posture monitoring logic and the generation and viewing logic of historical data visualization reports, which are referred to as monitoring logic and report logic in the following. In the monitoring logic, the process of dribbling training and posture monitoring is completed, and several data related to this training are automatically saved. The monitoring scope includes four common problems: frequent bowing, slow dribbling speed, dribbling wrist turning, and weak nondominant hand, and it provides corresponding feedback. According to the previous user research, the whole training process is shown in Figure 1 in the existing real training scene with coaching.

DribbleAid played part of the role of coach in this scene, such as finding problems and giving tips. According to this real scene, the logic of the user when using DribbleAid is sorted out to form a rough user scene, as shown in Figure 2.

According to user scenarios, a basic logical framework for training monitoring is designed. It is composed of equipment management process, training item and difficulty selection process, and posture problem monitoring process, as shown in Figure 3.

The entire device management process is composed of two small processes, namely, the device Bluetooth connection process and the device binding process. The device connection process and the device binding process are related to each other and are independent of each other. 


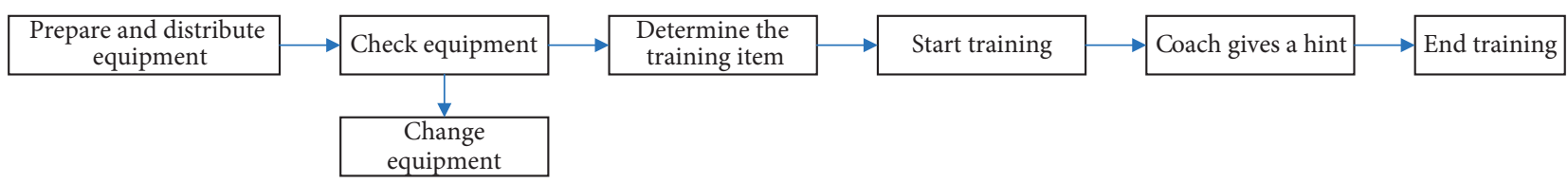

FIGURE 1: Real training scene with coaching.

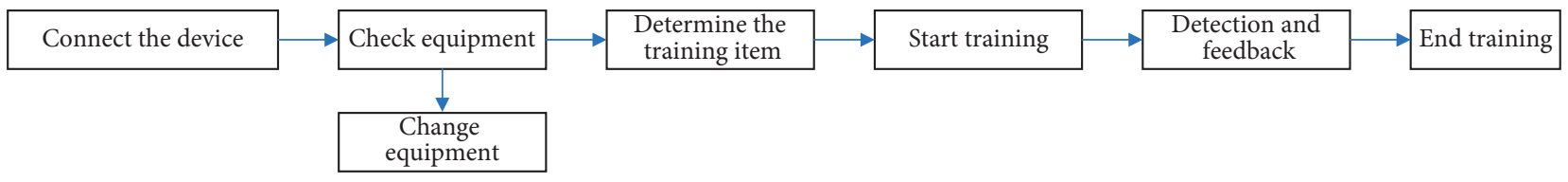

FIgURE 2: User scenario of DribbleAid.

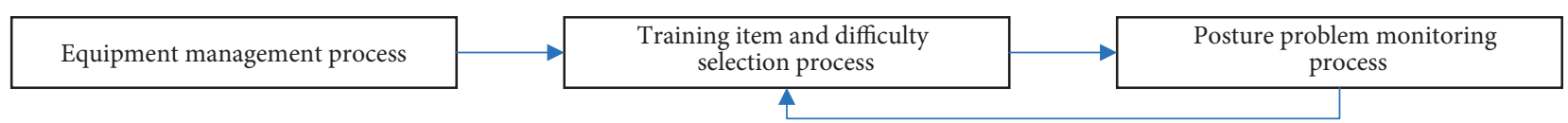

Figure 3: The basic logic framework of DribbleAid.

Device binding is used for the default device bundled with the native DribbleAid APP, which is generally the process used when DribbleAid is used for the first time. It can also be used when replacing equipment, such as after equipment is damaged and replaced with a new one. The Bluetooth connection process of the device is to connect the data transmission channel between the hardware device and the APP software through the Bluetooth module. Generally, it can be efficient and practical after the hardware is bound. When the device is not bound, Bluetooth can also be connected. However, it must be ensured that only one piece of DribbleAid hardware device is opened in the nearby environment. In the two processes of device management, the device Bluetooth connection process will be used every time the DribbleAid APP is enabled. The device binding only needs to be used when replacing a new device or using it for the first time. In addition, the device's Bluetooth connection process is more important to the DribbleAid APP, and the app is unavailable if you leave the device's Bluetooth connection process. The device binding process is a highly efficient process. After leaving, the APP will only be less efficient but still usable. According to the frequency of use and the importance of functions, the priority of the interaction logic is derived. The Bluetooth connection process of the device is greater than the device binding process.

The training item and difficulty selection process consists of two small processes: training item selection and difficulty selection. These two small processes jointly determine the selection process of training items for the rigorous degree of attitude monitoring. Training items here refer to the special training of different dribbling training content. Different training programs may have different standards for the same bad posture monitoring. For the four monitoring contents, each training item has its own set of monitoring standards. The difficulty selection process is another process that controls the strictness of the monitoring standards and is used in conjunction with the training selection process. The higher the difficulty, the stricter the monitoring standards under the same training item.

The posture problem monitoring process refers to the process of DribbleAid's posture monitoring during the user's dribble. In the process, the hardware part obtains the data, transmits the data to the mobile phone via Bluetooth, and determines whether to trigger the four problems to be monitored through a certain data model.

3.3. Related Software and Hardware Technology. Motion sensor hardware is the basis of DribbleAid implementation described in this study. The motion sensor module used in this study is TY61 six-axis motion sensor module produced by Shenzhen Vite Intelligent Co., LTD., equipped with Bluetooth, three-axis accelerometer, and gyroscope.

Step counting is a function widely used in sports bracelets and social apps recently. Its main content is realized by step counting algorithm. Step counting algorithm is an algorithm based on the accelerometer and based on the periodic change of the vertical acceleration value brought by the periodic change of the user's behavior. Its essence is to measure the times of the user's cyclic movement in accordance with a specific law and movement cycle. In this study, the idea of the periodic motion should also be applied to design an algorithm for measuring dribble frequency, whose essence is to measure the time taken by the user to complete several complete cycles of motion according to a specific law, and then the dribble frequency can be obtained.

Android is a free and open-source operating system based on Linux. This functional prototype is planned to be mounted on Android smartphones. This prototype will use Java language based on Linux to develop DribbleAid functional prototype software used on Android platform. 
(1) Initialize the quaternion Q. $q_{0}$ is the real part, $q_{1}, q_{2}$, and $q_{3}$ are the imaginary part, and $Q$ contains all the information of the attitude angle:

$$
\begin{aligned}
Q= & \left(\begin{array}{l}
q_{0} \\
q_{1} \\
q_{2} \\
q_{3}
\end{array}\right) \\
= & q_{0}+i q_{1}+j q_{2}+k q_{3} .
\end{aligned}
$$

(2) Obtain the measured values of angular velocity and acceleration, as well as magnetometer value; acceleration is $a x, a y$, and $a z$; angular velocity is $w x, w y$, and $w z$; magnetometer value is $m x, m y$, and $m z$.

(3) Convert the acceleration and magnetometer measurements into unit vectors, and obtain the gravity vector $\left(g_{x}, g_{y}, g_{z}\right)$ from the quaternion:

$$
\begin{aligned}
& g_{x}=\left[2\left(q_{1} q_{3}-q_{0} q_{2}\right)\right], \\
& g_{y}=\left[2\left(q_{2} q_{3}+q_{0} q_{1}\right)\right], \\
& g_{z}=\left[q_{0}^{2}-q_{1}^{2}-q_{2}^{2}+q_{3}^{2}\right] .
\end{aligned}
$$

(4) Calculate the error $\left(E_{a x}, E_{a y}, E_{a z}\right)$ :

$$
\begin{aligned}
& E_{a x}=a y * g z-a z * g y, \\
& E_{a y}=a z * g x-a x * g z, \\
& E_{a z}=a x * g y-a y * g x .
\end{aligned}
$$

(5) Using the error correction gyroscope, first obtain the accelerometer cumulative error ( $E_{a x}$ Int, $E_{a y}$ Int, $E_{a z}$ Int):

$$
\begin{aligned}
& E_{a x} I n t=E_{a x} I n t+E_{a x} \times K i, \\
& E_{a y} I n t=E_{a y} I n t+E_{a y} \times K i, \\
& E_{a z} I n t=E_{a z} I n t+E_{a z} \times K i .
\end{aligned}
$$

3.4. Prototype Development. In the process of prototype development, the following contents were researched and developed.

Through the basic data, extract relevant data information and complete the real-time monitoring function of "bow time is too long," "dribble turn wrist," and add the monitoring of state duration. Through the learning of step counting algorithm, the algorithm model of monitoring dribble frequency is completed, and the real-time monitoring function of "dribble speed is too slow" is realized.

The whole process is divided into 6 steps. The first step is to obtain the continuous angular acceleration basic data generated by the original hardware data. Step 2 records dribble cycle, and open-source project step algorithm is similar. Step 3 is to calculate the 3 consecutive dribbles; compared with the values specified, it can determine whether dribbling speed is too slow. In Step 4, for BUG modification process, the maximum angular acceleration of the minimum limit is reduced, and it is used to capture small dribbling actions. The fifth step shows the rule of dribbling speed judgment; every move will trigger a judgment. The sixth step uses time to free up space.

Complete the storage of dribble monitoring data and record the trigger time. Historical data can be easily viewed to support subsequent experiments.

Complete the real-time voice feedback function-when the bad posture is successfully monitored, the software will call the relevant prompt information to remind the user in real time. The author completed the voice recording in Baidu voice interface.

The modifiable parameters involved in the monitoring algorithm can be displayed on the software interface, and the modification of algorithm parameters can be completed on the interface, which is convenient for later experiments.

The pose input method of multisensory interaction is completed. "Start monitoring" instruction is to complete 5 consecutive dribbles. This is very natural, when connected to wear and connected to the hardware, just 5 dribbles directly can start monitoring, with no extra work. The "end of dribble" command, similar to the action of looking at a watch, continues $1 \mathrm{~s}$, meaning "it's almost time, have a rest."

During the test, it was also found that for the different monitoring angles of the two palms, the initial scheme was trying to divide the hardware device into three modules, left, right, and head, to wear hardware separately, which was specially responsible for the module part. As a result, there is always a wrist device idle during training, resulting in a waste of resources. Somatosensory interaction can solve this problem. Before sending the start command, join the process of confirming that "please confirm training hand" wrist valgus is more than 90 , which can be determined after holding for $1 \mathrm{~s}$, and then call the algorithm parameters of the corresponding hand.

\section{Experiments and Discussions}

4.1. Experiment Overview. The purpose of this experiment is to obtain the judgment conditions of DribbleAid monitoring function, namely, the monitoring standards mentioned above: Each set of standards includes the following judgment conditions: 1. Determine the determination condition of "bow for too long." 2. Determine the judgment condition of "dribble speed is too slow." 3. Determine the judgment conditions of "dribbling wrist flip." 4. Criteria for "weak nondominant hand." This determination is optionally added to the monitoring criteria for some projects. This study hopes to obtain the monitoring standards recognized by real coaches through this test, so that the problems monitored by DribbleAid can meet the judgment of real coaches.

The participating experts are 6 persons related to basketball education: 3 current or former coaches of the youth basketball training class and 3 professors of the college of physical education. Panel ONE includes three university professors specializing in physical education. The task of expert group I mainly lies in discussing and sorting out the overall idea of the experiment with the researchers before the experiment is carried out, judging whether the experimental 
idea sorted out by the researchers is feasible, and then communicating fully with the possible situations and matters for attention in the experiment. At the same time, the author needs to discuss with the expert members to develop a recognized parameter model for each monitoring point. Expert group 2 consists of three individuals concerned with basketball education. The task of expert group 2 mainly lies in observing the monitoring reminders of dribble players on-site and judging whether all the problem points monitored by DribbleAid conform to the coach's cognition based on their own teaching experience. If necessary, they can review the video for confirmation. The participants came from different levels of basketball enthusiasts, as well as students of a specific level recommended by the coach. The task of the subjects is to complete the training tasks assigned by the experiment, and each training time is no less than the prescribed time. The tasks of the subjects are relatively flexible and can be flexibly allocated according to the needs of the experiment.

\subsection{Evaluation on Head Bending Time and Dribble Turning} Wrist. When obtaining the judgment conditions of head bending time too long and dribble turning wrist, follow this concept: try different parameter combinations, and select the final parameter combination according to the recognition degree of the coach of the trigger condition monitored.

The basic design idea of Experiment 1 is as follows: 1. Set a relatively strict monitoring standard for "too long time of bowing head" and "turning wrist on dribble." The so-called relatively strict monitoring standards take "bow for too long" monitoring site as an example; the researchers will let a student wear DribbleAid headgear and let him try to bow, with realtime observation by the coach; the one-day coach thinks it is necessary to remind the students at this time. For example, at this time, the included angle between the surface of the student's head device and the horizontal plane is basically stable between $45^{\circ}$ and $60^{\circ}$, so $70^{\circ}$ will be selected as a relatively strict monitoring standard. 2. Please ask the coach to recommend several players of a certain level and conduct basketball dribbling training by simulating real situations. Meanwhile, use DribbleAid to monitor three young players for a period of time, and accept researchers to record the whole process of dribbling. 3. After dribbling, the number of starting problems at the monitoring point and the occurrence time recorded by the functional prototype as well as the video were handed over to the real basketball coach. Combined with the time of the occurrence of problems, the problem points monitored by the functional prototype were judged by reviewing the video, which were effective and invalid. The certification rule is that the minority is subordinate to the majority principle; that is, if more than half of the coaches think that the monitoring of a bad behavior is effective, the monitoring of the bad posture will be considered as effective; if only less than half of the coaches think that the monitoring of the bad posture is effective, the monitoring of the dark posture will be considered as invalid. 4 . Under the guidance of the coach, modify the monitoring standards and cycle the above process until the coach has a high recognition of the bad posture monitored by DribbleAid and there are no more omissions. 5. When the recognition reaches a high level and there is basically no missed judgment, the monitoring standards of "too long bowing time" and "dribble wrist flip" are selected as the general monitoring standards.

The two general monitoring criteria of "wrist flip on dribble" and "long bow time" were carried out in the scenario of "crossing in place or dribbling between legs with introductory difficulty." "Cross in place or dribble between the legs" was selected as the scene, because "too long time of bowing head" is a common problem in all scenes, while "dribble wrist flip" is a problem that is easily triggered when changing direction, and relevant data can be monitored more efficiently in this scene than in other scenes. The lowdifficulty scenario of "getting started" is also chosen because students with a lower level will make more mistakes and can monitor relevant data more efficiently. Because of the need for a large number of monitoring times, experts will designate capable people according to the need to randomly simulate the dribble error attitude prototype judgment.

After setting a relatively strict default value, iterate through the parameter values. The following is the parameter iteration process involved in the experiment. In the standard parameter iteration of the "dribble palm" test, the "motion sensor $X$-axis angle value threshold" and the "critical value exceeding continuous time" are the initial values. The three students tested will be monitored. Each student will dribble for no less than three minutes. Then the basketball assistants of the three camps will be invited to play back the video and record the occurrence time of the bad posture by the functional prototype. The captured bad posture will be certified separately.

When parameters are iterated, the minimum changes of "critical value of $X$-axis angle of motion sensor" and "duration exceeding critical value" are 5 degrees and $0.2 \mathrm{sec}$ onds, respectively. In addition, the coach participates in the process of modifying parameters.

Parameter iteration and relevant experimental data are shown in Table 1 and Figure 4 . It can be seen that group 4 has the highest recognition of each parameter combination. Group 5 and group 6 have a lot of omissions, but they are not used despite high recognition. The "red dots" in the line chart indicate that the monitoring based on this parameter combination will produce more omissions.

Finally, the optimal parameters of the monitoring standard of "dribbling wrist flip" are as follows: The critical value of $X$-axis angle of motion sensor is $105^{\circ}$; the duration of exceeding the threshold is 0.4 seconds.

If the downtime is too long, the process monitoring standard is 0 seconds. Taking 0 second as the initial value of " $X$-axis angle threshold of motion sensor" and "duration" exceeding the critical value, three students were monitored. Each student would have three minutes of dribble training time for testing. Then basketball assistants from three camps were invited to record the occurrence time of video playback and bad posture by functional prototype, and the captured bad posture was certified separately.

As found in the experimental process, "down time is too long" parameter to 5 degrees and 0.2 seconds for the 
smallest change, found that "the $X$-axis motion sensor Angle value threshold" and "duration" more than the critical value changes with the minimum value step by step, monitoring the results of different parameter combinations recognition ratio difference is not large, so parameter iteration of down time is too long; the minimum change of the data of " $X$-axis angle critical value of motion sensor" and "duration beyond critical value" is adjusted to 10 degrees and 0.5 seconds. Parameter iteration and relevant experimental data are shown in Table 2 and Figure 5. It can be seen that group 4 has the highest recognition for each parameter combination. Groups 5 and 6 have a few monitoring omissions, but they are given up despite high recognition. The "red dots" in the line chart indicate that the monitoring based on this parameter combination will produce more omissions, and the "bonus points" indicate that the monitoring based on this parameter combination will produce few omissions.

Finally, the optimal parameters of the monitoring standard of "long bow time" are as follows: The critical value of $X$-axis angle of motion sensor is $-120^{\circ}$; the duration of exceeding the threshold is 1 second.

4.3. Evaluation on Slow Dribbling Speed. According to 15 sets of monitoring standards, the coach selects 3 typical students in each standard, with each dribbling 99 times, and calculates the average dribble time of 3 dribbles as the reference value of the judgment condition of "dribble speed is too slow" in this monitoring standard. The basic idea of the second part of the experiment is as follows: 1 . Determine the training program and difficulty. 2. Ask the coach to recommend several "benchmark students" from all his students. The recommended rule is that the coach considers the dribble speed of the trainee to be the "slow dribble speed" monitoring standard for the training event and difficulty. 3. The researchers recorded 99 dribble times of all the "benchmark students" and calculated each dribble time of each student. 4. Find the average time. 5 . Then flexibly use the average time of each dribble to establish the monitoring standard of "slow dribble speed" under the training project and difficulty. Cycle the above process to determine the judgment condition of "slow dribbling rate" in all 15 sets of detection standards. In situ low hand dribble entry difficulty of the experimental process is an example. Please ask the coach to recommend three students who meet the recommended rules, and record the time of the 99 dribbles of the three students to find out the time for each student to dribble three times and the average time for each student to complete three dribbles, as shown in Figure 6. It is concluded that the monitoring standard of the entry difficulty of low hand dribble in situ is "the time spent for 3 consecutive complete dribbles is 1.83 seconds."

After repeating the above process, the pair ratios of all 15 judgment conditions of dribbling speed were obtained, as shown in Table 3. The data with "*" in the table appears because the average time of one student is obviously
TABLE 1: The iteration of Reversal's monitoring basis.

\begin{tabular}{lccccc}
\hline Group & $\begin{array}{c}\text { Parameter } \\
\text { combination }\end{array}$ & Number & $\begin{array}{c}\text { Approval } \\
\text { number }\end{array}$ & $\begin{array}{c}\text { Proportion } \\
(\%)\end{array}$ & Error \\
\hline 1 & $90^{\circ}-0 \mathrm{~s}$ & 147 & 29 & 19.73 & No \\
2 & $100^{\circ}-0.2 \mathrm{~s}$ & 45 & 22 & 48.89 & No \\
3 & $105^{\circ}-0.2 \mathrm{~s}$ & 39 & 21 & 53.85 & No \\
4 & $105^{\circ}-0.4 \mathrm{~s}$ & 37 & 28 & 75.68 & No \\
5 & $110^{\circ}-0.4 \mathrm{~s}$ & 7 & 7 & 100 & More \\
6 & $105^{\circ}-0.6 \mathrm{~s}$ & 12 & 12 & 100 & More \\
\hline
\end{tabular}

lower than that of the other two, so the number is taken as the average of the other two.

4.4. Evaluation on Weak Nondominant Hand. In experiment 3 , the coach selected a number of typical students to complete the training of the same difficulty and item with the left and right hands and added the functional prototype of the judgment condition of the previous two experiments to monitor the posture. By comparing the trigger times of the same monitoring points with the left and right hands, the judgment condition of "weak nondominant hand" was determined.

The third part of the experimental ideas is as follows: 1 . Please ask the coach to recommend several trainees who have mild "weak nondominant hand" and have a certain level. 2. The left and right hands were trained with low difficulty in situ dribbling, and the DribbleAid functional prototype, which has imported 15 sets of monitoring standards, was used to monitor them. 4. In case of occurrence, coaches are requested to assist in formulating the judgment condition of "weak nondominant hand." In this part, the concept of "trigger rate of monitoring point" will be introduced, which refers to the number of triggers per minute of a monitoring point. For example, a training lasts for 2 minutes, among which "bow time is too long" triggers 10 times, "dribble speed is too slow" triggers 4 times, and "dribble turns wrist" starts 3 times. The trigger rates of the three are 5 times/min, 2 times/min, and 1.5 times $/ \mathrm{min}$, respectively.

Please ask the coach to recommend 3 cadets who have certain dribbling skills and mild "weak nondominant hand" problem. Under the medium difficulty condition of dribbling with low hand in place, 3 subjects trained with DribbleAid functional prototype for 2 minutes, and the test results were obtained, as shown in Table 4 and Figure 7.

After discussion with experts, it is concluded that the triggering conditions of "weak nondominant hand" are as follows: After the training, the trigger rate of any two monitoring points was 1.2 times higher than that of the corresponding monitoring points in the latest training of low hand dribbling. In addition, after the training of low hand dribbling in situ, in the three movements of "too long time on the ground," "too slow dribbling speed," and "palm dribbling," the trigger rate of any two movements is higher than that of the other hand in the 12 corresponding tests of the latest training of low hand dribbling in situ, that is, "weak non-dominant hand" and voice reminder. 


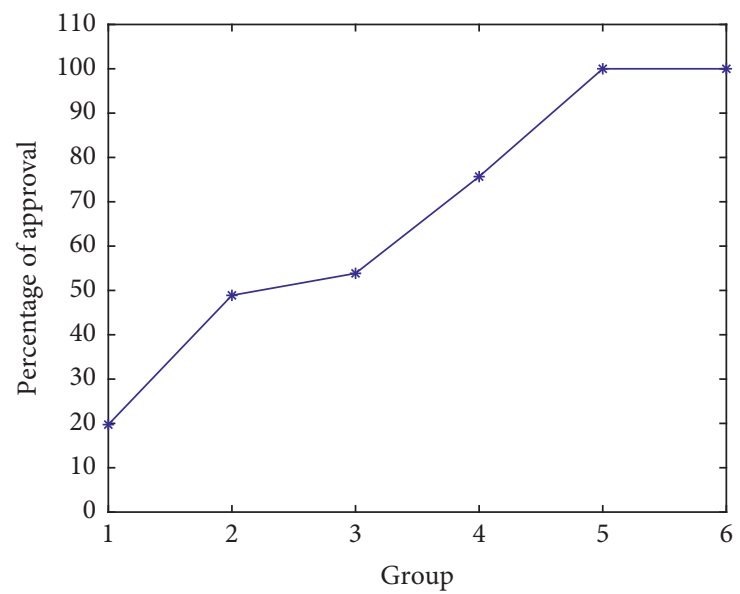

FIGURE 4: Iterative process of the combination of dribbling and wrist-turning parameters.

TABLE 2: The iteration of Bow's monitoring basis.

\begin{tabular}{|c|c|c|c|c|c|}
\hline Group & Parameter combination & Number & Approval number & Proportion (\%) & Error \\
\hline 1 & $-100^{\circ}-0.5 \mathrm{~s}$ & 287 & 69 & 24.04 & No \\
\hline 2 & $-100^{\circ}-1.0 \mathrm{~s}$ & 132 & 70 & 53.03 & No \\
\hline 3 & $-110^{\circ}-1.0 \mathrm{~s}$ & 93 & 65 & 69.89 & No \\
\hline 4 & $-120^{\circ}-1.0 \mathrm{~s}$ & 81 & 75 & 92.59 & No \\
\hline 5 & $-130^{\circ}-1.0 \mathrm{~s}$ & 42 & 40 & 95.24 & More \\
\hline 6 & $--120^{\circ}-1.5 \mathrm{~s}$ & 42 & 39 & 92.86 & More \\
\hline
\end{tabular}

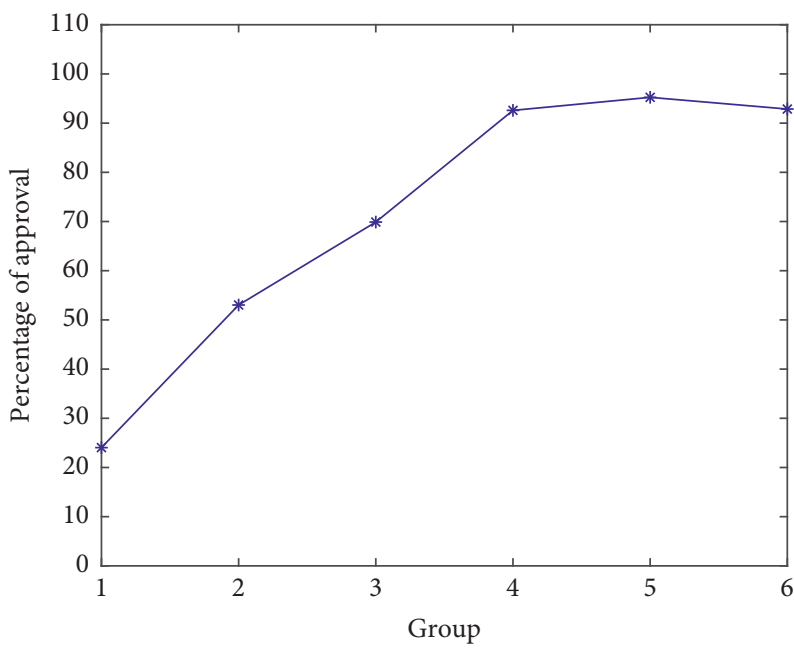

FIgURE 5: Iterative process of parameter combination for too long bowing time. 


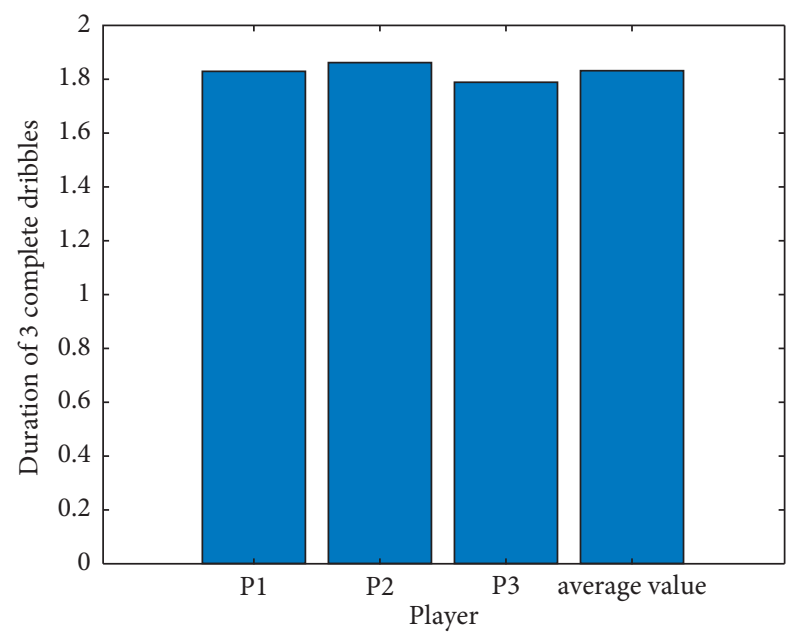

Figure 6: The time and average of 3 dribbles for 3 trainees.

Table 3: All of the monitoring basis of Dribble speed.

\begin{tabular}{|c|c|c|c|c|c|}
\hline Item & Low hand dribbling & Crossover dribble & Crossovers & Behind the dribble & Hands dribbling \\
\hline Introduction & 1.73 & 1.88 & 2.06 & 2.09 & 2.06 \\
\hline Medium & 1.34 & 1.29 & 1.11 & $1.42^{*}$ & 1.31 \\
\hline Challenge & 1.17 & 1.19 & $1.05^{*}$ & $1.22^{*}$ & 1.14 \\
\hline
\end{tabular}

Table 4: Data of 3 examinees on both hands.

\begin{tabular}{|c|c|c|c|c|}
\hline Personnel & Project & Wrist-flipping trigger rate & Rate trigger rate & Bow trigger rate \\
\hline \multirow{3}{*}{$P 1$} & Left (times/min) & 2.9 & 3.5 & 5.5 \\
\hline & Right (times/min) & 2.3 & 2.6 & 4.4 \\
\hline & Ratio & 1.26 & 1.35 & 1.25 \\
\hline \multirow{3}{*}{$P 2$} & Left (times/min) & 1.4 & 3.3 & 6.2 \\
\hline & Right (times/min) & 1.6 & 1.8 & 4.3 \\
\hline & Ratio & 0.88 & 1.83 & 1.44 \\
\hline \multirow{3}{*}{$P 3$} & Left(times/min) & 1.5 & 4.7 & 6.3 \\
\hline & Right(times/min) & 0.4 & 2.8 & 2.9 \\
\hline & Ratio & 3.75 & 1.68 & 2.17 \\
\hline
\end{tabular}

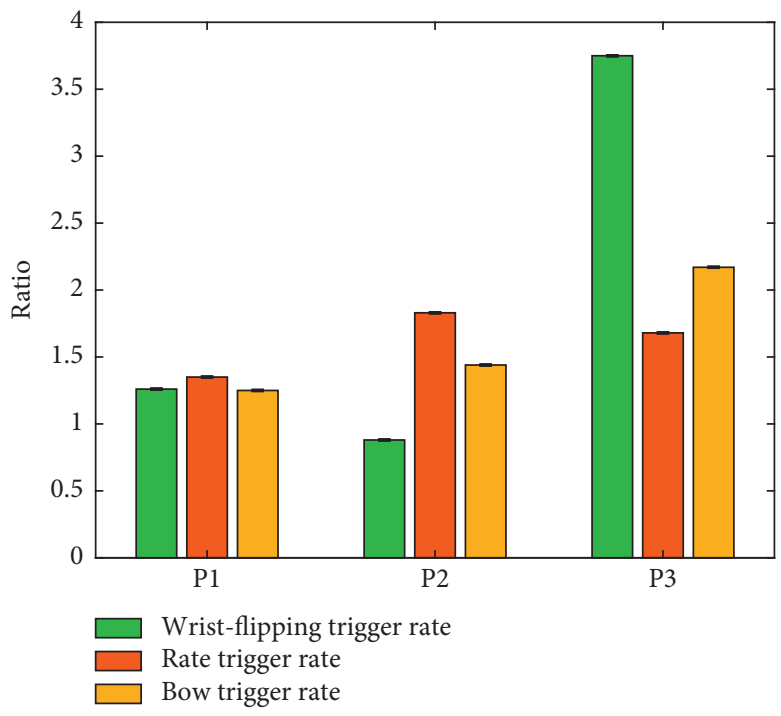

FIgURE 7: The testing ratio of three players. 


\section{Conclusions}

The purpose of this study is to seek a feasible solution for the outstanding problem in basketball dribble teaching of grassroots physical education in China, which is caused by the low teacher-student ratio and the students' inability to obtain adequate guidance. The highlight of this study, sufficient user research and the experiment of obtaining monitoring standards, is the key part of all the issues involved in this study, and its conclusion is the biggest innovation point of this study. The user research part including the basic school teachers and students in the teaching process of pain points research pointed out the direction of the product and the experts to determine the product function of the interview, and the user trial of the high-fidelity prototype is the most intuitive embodiment of the DribbleAid product solution function effectiveness and concept design of the user evaluation. This part provides the most fundamental basis for DribbleAid development. In the experimental part of obtaining the monitoring standard, the posture monitoring standard that conforms to the teaching experience and cognition of real coaches is extracted, which is the fundamental guarantee of the functional effectiveness of DribbleAid.

\section{Data Availability}

The datasets used during the current study are available from the corresponding author upon reasonable request.

\section{Conflicts of Interest}

The authors declare that there are no conflicts of interest.

\section{References}

[1] J. L. Fox, C. J. O'Grady, and A. T. Scanlan, "The relationships between external and internal workloads during basketball training and games," International Journal of Sports Physiology and Performance, vol. 15, no. 8, pp. 1081-1086, 2020.

[2] A. J. Petway, T. T. Freitas, J. Calleja-González, D. Medina Leal, and P. E. Alcaraz, "Training load and match-play demands in basketball based on competition level: a systematic review," PLoS One, vol. 15, no. 3, 2020.

[3] J.-G. Wang, K.-L. Cai, Z.-M. Liu et al., "Effects of minibasketball training program on executive functions and core symptoms among preschool children with autism spectrum disorders," Brain Sciences, vol. 10, no. 5, 263 pages, 2020.

[4] F. D'Elia, D. D'Andrea, G. Esposito, G. Altavilla, and G. Raiola, "Increase the performance level of young basketball players through the use of high intensity interval training," International journal of human movement and sports sciences, vol. 9, no. 3, pp. 445-450, 2021.

[5] M. Reina, J. García-Rubio, and S. J. Ibáñez, "Training and competition load in female basketball: a systematic review," International Journal of Environmental Research and Public Health, vol. 17, no. 8, 2639 pages, 2020.

[6] J. Pei, K. Zhong, J. Li, J. Xu, and X. Wang, "Ecnn: Evaluating a cluster-neural network model for city innovation capability," Neural Computing \& Applications, pp. 1-13, 2021.

[7] M. Lastella, G. D. Roach, G. E. Vincent, A. T. Scanlan, S. L. Halson, and C. Sargent, "The impact of training load on sleep during a 14-day training camp in elite, adolescent, female basketball players," International Journal of Sports Physiology and Performance, vol. 15, no. 5, pp. 724-730, 2020.

[8] E. B. Asher, N. Panda, C. T. Tran, and M. Wu, "Smart wearable device accessories may interfere with implantable cardiac devices," HeartRhythm Case Reports, vol. 7, no. 3, pp. 167-169, 2021.

[9] M. Periša, T. M. Kuljanić, I. Cvitić, and P. Kolarovszki, "Conceptual model for informing user with innovative smart wearable device in industry 4.0," Wireless Networks, vol. 27, no. 3, pp. 1615-1626, 2021.

[10] A. I. Faisal, S. Majumder, R. Scott, T. Mondal, D. Cowan, and M. J. Deen, "A simple, low-cost multi-sensor-based smart wearable knee monitoring system," IEEE Sensors Journal, vol. 21, no. 6, pp. 8253-8266, 2020.

[11] M. Zhu and H. Pham, "An empirical study of factor identification in smart health-monitoring wearable device," IEEE Transactions on Computational Social Systems, vol. 7, no. 2, pp. 404-416, 2020.

[12] N.-W. Lo and A. Yohan, "BLE-based authentication protocol for micropayment using wearable device," Wireless Personal Communications, vol. 112, no. 4, pp. 2351-2372, 2020.

[13] E. Park, "User acceptance of smart wearable devices: an expectation-confirmation model approach," Telematics and Informatics, vol. 47, Article ID 101318, 2020.

[14] J. Li, N. Zhang, J. Ni, J. Chen, and R. Du, "Secure and lightweight Authentication with key agreement for smart wearable systems," IEEE Internet of Things Journal, vol. 7, no. 8, pp. 7334-7344, 2020.

[15] S. Nižetić, N. Pivac, V. Zanki, and A. M. Papadopoulos, "Application of smart wearable sensors in office buildings for modelling of occupants' metabolic responses," Energy and Buildings, vol. 226, Article ID 110399, 2020.

[16] H.-K. Wang, C.-H. Chen, T.-Y. Shiang, M.-H. Jan, and K.-H. Lin, "Risk-factor Analysis of high school basketballplayer ankle injuries: a prospective controlled cohort study evaluating postural sway, ankle strength, and flexibility," Archives of Physical Medicine and Rehabilitation, vol. 87, no. 6, pp. 821-825, 2006.

[17] M. Reina, J. García-Rubio, P. T. Esteves, and S. J. Ibáñez, "How external load of youth basketball players varies according to playing position, game period and playing time," International Journal of Performance Analysis in Sport, vol. 20, no. 6, pp. 917-930, 2020.

[18] F. M. Verhoeven and K. M. Newell, "Coordination and control of posture and ball release in basketball free-throw shooting," Human Movement Science, vol. 49, pp. 216-224, 2016.

[19] S. Forghany, S. B. Dehkordi, H. M. Sanech, and M. Mashhadi, "Foot posture in basketball players with history of the shin splint," Journal of Research in Rehabilitation Sciences, vol. 9, no. 3, pp. 406-414, 2013.

[20] P. Esteves, "Postura corporal e relações angulares face ao cesto influenciam a tomada de decisão no 1v1 em basquetebol," Cuadernos de Psicología del Deporte, vol. 12, no. 1, pp. 17-22, 2012.

[21] M. Knoblauch, "The History of Wearable Tech, from the Casino to the Consumer," Mashable com, vol. 45, 13 pages, 2014.

[22] E. O. Thorp, "The invention of the first wearable computer," in Proceedings of the Digest of papers. Second International Symposium on Wearable Computers, pp. 4-8, Pittsburgh, PA, USA, October 1998. 
[23] J. L. Mudge, J. W. Zimmer, and K. G. Taft, "V-groove isolated integrated circuit memory with integral pinched resistors," U.S. Patent 3,979,612[P]. 1976-9-7, 1976.

[24] E. Margolies, Which way did He go: the private Eye in Dashiell Hammett, Raymond Chandler, Chester Himes, and Ross Macdonald, Holmes \& Meier Pub, Teaneck, NJ, USA, 1982.

[25] D. Kendrick, "A reading machine in your pocket," introducing the KNFB reader mobile edition, vol. 32, no. 2, pp. 10-19, 2008.

[26] S. Mann, "Wearable computing: a first step toward personal imaging," Computer, vol. 30, no. 2, pp. 25-32, 1997.

[27] Z. He, T. Chang, S. Lu, H. Ai, D. Wang, and Q. Zhou, "Research on human-computer interaction technology of wearable devices such as augmented reality supporting grid work," Procedia Computer Science, vol. 107, pp. 170-175, 2017.

[28] E. Mencarini, A. Rapp, L. Tirabeni, and M. Zancanaro, "Designing wearable systems for sports: a review of trends and opportunities in human-computer interaction," IEEE Transactions on Human-Machine Systems, vol. 49, no. 4, pp. 314-325, 2019.

[29] A. Adapa, F. F.-H. Nah, R. H. Hall, K. Siau, and S. N. Smith, "Factors influencing the adoption of smart wearable devices," International Journal of Human-Computer Interaction, vol. 34, no. 5, pp. 399-409, 2018.

[30] A. Schmidt, H. W. Gellersen, and C. Merz, "Enabling Implicit Human Computer Interaction: A Wearable RFID-Tag Reader," in Proceedings of the Digest of Papers. Fourth International Symposium on Wearable Computers, pp. 193-194, Atlanta, GA, USA, October 2000.

[31] M. Antona and C. Stephanidis, "Universal access in humancomputer interaction," in Proceedings of the Access to the Human Environment and Culture: 9th International Conference, pp. 2-7, Los Angeles, CA, USA, August, 2015. 